become seizure free within 3 months of initiating the $\mathrm{KD}$ have an excellent long-term outcome. (Jung DE, Kang HC, Kim HD. Long-term outcome of the ketogenic diet for intractable childhood epilepsy with focal malformation of cortical development. Pediatrics August 2008;122:e330-e333). (Respond: Heung Dong Kim MD, PhD, Yonsei University College of Medicine, Brain Research Institute, Severance Children's Hospital, 134 Shinchondong, Seodaemun-gu, Seoul 120-752, Korea. E-mail: hdkimmd(a)yuhs.ac).

COMMENT. The ketogenic diet (KD) was first introduced for the treatment of epilepsy at the Mayo Clinic (Wilder RM. Mayo Clin Bull 1921;2:307). Unlike the later Johns Hopkins protocol (Livingston S. Postgrad Med 1951;10:333-336; Freeman JM et al. Pediatrics 1998;102:158-1363), the classic Mayo KD is introduced without initial fasting (NFKD) and usually, without admission to hospital. In my experience using the NFKD, a smaller ratio of ketogenic to antiketogenic items than that employed by the present authors has usually been successful in younger children, the higher $4: 1$ ratio being necessary only in older children (Millichap JG et al. Am J Dis Child 1964;107:593-604; JAMA 1966;198:210). In a Korean multicenter study involving 199 patients, a comparison of the modified Mayo non-fasting KD and the Hopkins fasting KD, found that by omitting the fasting period, especially in young children, acute dehydration was prevented, with no difference in the time to ketosis or in the efficacy of the diet. However, by employing the relatively high $4: 1$ ratio, favored by the Hopkins method, serious adverse effects were not avoided, including 5 deaths related to lipoid aspiration pneumonia, serious infection, and nutritional problems. (Kang HC et al. Epilepsia 2005;46:272-279; Ped Neur Briefs Feb 2005:19:12-13).

\title{
RISK OF MORTALITY IN CHILDREN WITH FEBRILE SEIZURES
}

Mortality after febrile seizures was studied in a large population-based cohort of children in Denmark followed from 3 months of age up to 25 years or until death, by researchers at Institute of Public Health, and National Centre for Register-based Research, Aarhus University; and University Hospital, Aarhus, Denmark; and School of Public Health, UCLA, USA. Of 1.6 million children born between 1977 and 2004, 8172 died, including 232 deaths in 55,215 children with a history of febrile seizures. The mortality rate ratio (1.80) was $80 \%$ higher during the first year and $90 \%$ higher (1.89) during the second year after the first febrile seizure; 132 of 100,000 children died within 2 years of a febrile seizure compared with 67 deaths per 100,000 without a history of febrile seizures. The increase in mortality (rate ratio 1.99) was restricted to patients with complex febrile seizures $(>15 \mathrm{~min}$ or recurrence within $24 \mathrm{hr}$ ); children with simple febrile seizures $(<15 \mathrm{~min}$ and no recurrence within $24 \mathrm{hr}$ ) had a mortality rate similar to the background population (rate ratio 1.09). The cause-specific cumulative mortality within 2 years of a febrile seizure per 100,000 children was 13 for seizures, 11 for pneumonia, 11 for sudden unexpected death, and 11 for congenital malformation of the nervous system. The development of epilepsy was not the explanation for increased mortality in all cases. The risk of sudden unexpected death was five times greater during the 2 years after a first febrile seizure than in the background population. (Vestergaard M, Pedersen MG, Ostergaard JR, Pedersen CB, Olsen J, Christensen J. Death in children with febrile seizures: a population-based cohort study. Lancet Aug 9, 2008;372:457-463). (Respond: E-mail: mogens.vestergaard( $u$ alm.au.dk). 
COMMENT. An increased risk of mortality in children with a history of febrile seizures is restricted to patients with complex febrile seizures. The risk though significant is small, but the cause is not completely understood. The occurrence of epilepsy or neurologic abnormality may be contributory in some but not in all deaths. Mazumdar $M$ in a commentary (Lancet 2008;372:429-430) recommended further follow-up of complex cases. Kinney H et al. (Pediatr Dev Pathol 2007;10:208-223) relates sudden death in 5 toddlers with febrile seizures to developmental hippocampal pathology and possible nocturnal seizures. A shared susceptibility to SIDS and febrile seizures has not been proven. That febrile seizures may be less benign than generally assumed is suggested by the above study and also by the recent report of MRI abnormalities in patients with first simple or complex febrile seizures. (Hesdorffer DC et al. Epilepsia 2008;49:765-771). In practice, MRI is not indicated for the evaluation of simple febrile seizures, but neurologic consultation is advisable in children with recurrent febrile seizures and in those with complex seizures. (Ped Neur Briefs June 2008;22:47-48).

Omega-3 fatty acids recommended in treatment of refractory seizures and in prevention of sudden unexpected death in epilepsy. (Scorza FA et al. Epilepsy Behav Oct $2008 ; 13: 279-283)$. The recommendation is based on clinical and animal studies that have demonstrated anticonvulsant properties of omega-3 supplementation and a reduction in cardiac arrhythmias and sudden cardiac deaths, the proposed mechanism of sudden death in epilepsy.

\section{SPECTRUM OF BENIGN OCCIPITAL EPILEPSIES OF CHILDHOOD}

The clinical, electroencephalographic and genetic characteristics of Panayiotopoulos and Gastaut syndromes were explored, using twin and multiplex family studies, by researchers at The University of Melbourne, Australia, and Tel Aviv University, Israel. Sixteen probands including 7 twins were classified into early, late or mixed benign occipital epilepsy of childhood (BOEC) syndromes, and 9 non-twin probands with a family history of epilepsy were included. One-third of the children in this selected series of BOEC had a mixed syndrome with features of both Panayiotopoulos (early) and Gastaut (late) syndromes. Monozygotic twin pairs had a similar concordance rate to that of dizygotic twin pairs, suggesting that BOEC may not be a purely genetic disorder. A mixed pattern of focal and generalized epilepsies, mainly focal, was found in relatives with epilepsy. Panayiotopoulos and Gastaut syndromes are not distinct genetic entities, but part of an electro-clinical BOEC spectrum, with shared genetic and environmental determinants. (Taylor I, Berkovic SF, Kivity S, Scheffer IE. Benign occipital epilepsies of childhood: clinical features and genetics. Brain September 2008;131:2287-2294). (Respond: Prof Ingrid E Scheffer, Epilepsy Research Centre, Austin Health, West Heidelberg, Victoria 3081, Australia. E-mail: scheffer(a)unimelb.edu.au).

COMMENT. The syndromes of Panayiotopoulos and Gastaut are not distinct, but part of a continuum of BOEC syndromes, with overlapping electro-clinical features, and largely undetermined, shared genetic and environmental factors in etiology. SCN1A missense mutation, identified with generalized epilepsy with febrile seizures plus and Dravet 